\title{
The Potential of Neurofilament Light as a Biomarker in Alzheimer's Disease
}

\author{
Yong-lan Xiong a Tao Meng ${ }^{b}$ Jing Luo ${ }^{a}$ Hua Zhang ${ }^{a}$ \\ aDepartment of Neurology, The First Affiliated Hospital of Chongqing Medical University, Chongqing, China; \\ ${ }^{b}$ Department of Neurology, Chongqing University Central Hospital, Chongqing, China
}

\section{Keywords}

Neurofilament light · Alzheimer's disease · Biomarkers ·

Cognitive function · Neuroimaging

\begin{abstract}
Background: Alzheimer's disease (AD) is the most common neurodegenerative disease characterized by progressive memory loss and cognitive impairment. In 2011, the National Institute on Aging and Alzheimer's Association (NIA-AA) Research Framework has proposed to use biomarkers to diagnose $A D$ in living persons. AD core biomarkers show high diagnostic specificity in distinguishing AD from healthy control subjects, but have little additional value for prognosis or stage of disease. Summary: With the update of detection methods and techniques, other AD biomarkers have been discovered. Neurofilament light (NFL) is currently recognized as a biomarker of nerve axonal injury and one of the candidate markers in $A D$ neurodegeneration, and the relationship between NFL and AD pathophysiology has attracted widespread attention. More and more studies have shown that NFL plays an important role in predicting the clinical progress and prognosis of AD. Recently, the genomewide association study also found that multiple single-nu-
\end{abstract}

karger@karger.com

(C) 2021 S. Karger AG, Basel

www.karger.com/ene

Karger! cleotide polymorphisms are associated with NFL levels and AD risk. Key Messages: In this review, we discuss the relationship between the genetic characteristics of NFL and AD, the NFL levels in $A D$, and the relationship between NFL and $A D$ core biomarkers, neuroimaging, and cognitive performance.

(c) 2021 S. Karger AG, Basel

\section{Introduction}

Neurofilament light (NFL) chain is the most abundant intermediate filament protein in myelinated subcortical axons, which plays an important role in the assembly and maintenance of axonal cytoskeleton [1]. Axonal injury or neuronal degeneration releases NFL into interstitial fluid and eventually into cerebrospinal fluid (CSF) and plasma [2]. The level of NFL is increased in a variety of neurodegenerative diseases, including frontotemporal dementia (FTD), vascular dementia (VaD), and Alzheimer's disease (AD) [3]. According to the 2018 National Institute on Aging and Alzheimer's Association (NIA-AA) Research Framework on biological AD, NFL, as one of the candidate biomarkers of $(\mathrm{N})$ group, is not specific for 
neurodegeneration due to $\mathrm{AD}$. However, neurodegeneration, particularly synapse loss, is the aspect of AD neuropathologic change that correlates most closely with symptoms. Therefore, NFL provides important pathological staging information, which means NFL can be a biomarker in AD, but not a biomarker of AD [4]. Studies have found that CSF NFL is associated with the severity and survival of $\mathrm{AD}[5]$.

The collection of CSF through lumbar puncture is invasive, and longitudinal analysis has been rare, hindering the wider clinical application of NFL. In contrast, plasma samples are easier to obtain and less invasive, and some studies have reported a good correlation between plasma NFL and CSF NFL $[6,7]$. Therefore, reliable quantification of NFL in the plasma will be a big step toward biomarkers of the neurodegenerative process. However, the concentration of NFL in plasma is much lower than that in CSF, and $>50 \%$ of plasma samples cannot be reliably quantified by the electrochemiluminescence (ECL) test and enzyme-linked immunosorbent assay (ELISA) [8]. Single molecule array (SIMOA) is the most mature ultrasensitive technology in the field of blood-derived biomarkers for $\mathrm{AD}$ pathology. In terms of quantitative NFL, the SIMOA platform is 126 and 25 times more sensitive than ELISA and ECL, respectively. The NFL in all plasma samples is within the measurable range of SIMOA, thus providing the feasibility of quantitative determination of NFL in plasma [8]. At the same time, continuous measurement of plasma samples makes it possible to longitudinally analyze the levels of NFL. This article reviews the effects of genetic factors on NFL levels and AD risk and the current status of NFL levels in AD studies and summarizes the predictive role of NFL in clinical progression and neurodegeneration of $\mathrm{AD}$.

\section{The Research History of NFL in AD}

Neurofilaments (NFs) are neuron-specific intermediate filaments composed of 4 subunits (NFL, NF medium [NFM], and NF heavy [NFH] chain and $\alpha$-interexin) [1]. They are highly expressed in dendrites, neuronal cell bodies, and axons and play an important role in structural support, transport, and nerve transmission [9]. The destruction of the axon membrane releases NF into the interstitial fluid. Among the 4 subunits, NFL is the most abundant and soluble, which makes NFL the most measurable NF subunit in biological fluid [1]. One of the earliest studies found that abnormal accumulation of NF can cause motor neuron degeneration [10]. In 1996, a cross-

The Potential of NFL as an AD Biomarker sectional study found that the concentration of NFL in CSF increased in patients with amyotrophic lateral sclerosis (ALS), especially in patients with pyramidal tract involvement, and was also characteristic of $\mathrm{AD}, \mathrm{VaD}$, and normal pressure hydrocephalus, but the increase was smaller than that in ALS patients. The authors concluded that CSF NFL is a promising biomarker of neurodegeneration [11]. In 2014, a cross-sectional study of 3,356 patients with dementia found that CSF NFL differed among clinical diagnoses, with the highest levels seen in FTD, $\mathrm{VaD}$, and mixed $\mathrm{AD}$ and $\mathrm{VaD}$. Early-onset $\mathrm{AD}$ (younger than 65 years) had the lowest levels. High CSF NFL correlated with low MMSE score and short survival time irrespective of diagnosis and was also particularly evident in $\mathrm{AD}$ [5]. Researchers began to pay attention to the correlation between NFL and AD pathology. In a basic experiment, transgenic amyloid precursor protein or presenilin-1 (APP/PS1) mice established under the background of NFL knockout were used as subjects. It was found that NFL deficiency significantly increased cortical dystrophy axon pathology, amyloid $\beta$ (A $\beta$ ) deposition, synaptic fragility, and microglial proliferation around $A \beta$ plaques, suggesting that NFL may play a protective role in $\mathrm{AD}$ pathology [12]. Iron levels in the brain and sciatic nerve increased in NFL knockout mice. Changes in NFs during brain aging may cause excessive iron in the intracellular or extracellular environment, which may increase the risk of neuronal degeneration or protein accumulation in neurodegenerative diseases such as AD [13].

\section{The Relationship between the Genetic Characteristics of NFL and AD}

Through genome-wide association study (GWAS), it was found that 2 single-nucleotide polymorphisms (SNPs) (rs465401 and rs460420) located near the ADAMTS1 (a disintegrin-like and metalloproteinase with thrombospondin type 1 motif) gene on chromosome 21 were significantly associated with NFL in CSF. It is suggested that rs465401 and rs460420 may be the regulators of CSF NFL levels. Since rs465401 was most strongly associated with NFL in CSF, further survival analysis of rs465401 showed that rs465401 was associated with the risk of prodromal $\mathrm{AD}$ in normal cognitive participants during follow-up and that the risk of clinical progression in normal AA homozygotes was $>3$ times that of GG homozygotes during follow-up [14].

The small allele (T) of rs7943454 located in the leucine zipper protein 2 (LUZP2) region of chromosome 11p14.3 
was dose-dependent with higher plasma NFL levels. On the contrary, the small allele $(G)$ of rs640476 near gamma-aminobutyric acid type A receptor beta- 2 subunit on chromosome $5 \mathrm{q} 34$ was dose-dependent in all diagnostic groups except the mild cognitive impairment (MCI) group. In the longitudinal study, the rs7943454 small allele $(\mathrm{T})$ in LUZP2 increased the risk of AD (odds ratio = $1.547,95 \% \mathrm{CI}=1.018-2.351)$ and was associated with atrophy of the right temporal gyrus in the entire cohort $(p=0.0234)$. Using plasma NFL as the GWAS internal phenotype of $\mathrm{AD}$, it can be determined that rs7943454 is a new $\mathrm{AD}$ risk site [15].

Another study found that 3 SNPs (rs16840041, rs2269714, and rs2269715) located in the CD1A region of chromosome 1q23.1 were significantly correlated with the rate of increase in plasma NFL levels. Rs16840041-A can promote the progression of clinical diseases. Compared with the AA/AG group, the estimated time to disease progression in the GG group was significantly longer. In the Cox regression model (adjusted for age, diagnosis, and apolipoprotein 4 status), individuals with AA/ $\mathrm{AG}$ genotypes have a higher risk of developing $\mathrm{AD}$ [16].

However, it is not clear how these SNPs affect NFL levels. It is worth noting that these study participants were from the Alzheimer's Disease Neuroimaging Initiative (ADNI) cohort and that genetic variations associated with CSF and plasma NFL levels occurred on different chromosomes, and given the correlation between CSF and plasma NFL levels, further research is needed to determine whether the SNP variants on these chromosomes affect both CSF and plasma NFL levels. Since the participants in these studies are limited to non-Hispanic white participants, there may be race-dependent effects, and whether these variants have the same impact on NFL levels and $\mathrm{AD}$ risk in other populations needs further research.

\section{The NFL Levels in AD}

\section{The NFL Levels in Late-Onset AD}

Cross-sectionally, plasma NFL levels showed an increasing trend between the control group, MCI group, and AD group [17, 18]. Similarly, the level of CSF NFL also showed an increasing trend between the diagnosis groups. The negative correlation between the baseline Mini-Mental State Examination (MMSE) score and the NFL level further supports this view [19]. In the MCI group, there was no significant difference in plasma NFL levels between the $A \beta$-positive progression group and the
$\mathrm{A} \beta$-positive stable group. However, the stable MCI was defined based on a short follow-up period ( 2 years). This period may be too short to verify the benign nature of the so-called stable conditions. There was no difference in plasma NFL between the $A \beta$-positive control and $A \beta$ negative control, suggesting that any neuronal damage that may occur in the $A \beta$-positive control (i.e., preclinical $\mathrm{AD}$ ) is below the detection limit of plasma NFL [20]. In general, the differences in cross-sections are only apparent in the MCI and dementia stages.

Longitudinally, a cross-sectional study of the Kerr Anglican Retirement Village Initiative in Aging Health cohort found that changes in plasma NFL began to appear at the end of preclinical AD [21]. A study of 243 cognitively normal (CN) participants from the ADNI database also found that plasma NFL concentration and its rate of change were already abnormally high in the preclinical phase of $\mathrm{AD}$ [22]. A recent study demonstrated that 13 years before diagnosis, plasma NFL absolute levels in participants who developed $\mathrm{AD}$ were lower than those in the control group who maintained a dementiafree age and sex match during follow-up, but plasma levels in patients who developed $\mathrm{AD}$ began to deviate from those in the control group 10 years before diagnosis. Although there was a linear increase in plasma NFL in both $\mathrm{AD}$ patients and the control group, the change of NFL per year was 3.4 times faster for participants who developed AD dementia ( $\log _{2} 0.04 \mathrm{pg} / \mathrm{mL}$ [95\% CI: 0.02-0.06]) compared to those who did not $\left(\log _{2} 0.01 \mathrm{pg} / \mathrm{mL}\right.$ [95\% CI: $0.00-0.02$ ], $p$ for interaction $<0.0001$ ). They found that the effect of time on the rate of change of plasma NFL was not statistically significant, that is, changes in plasma levels of NFL did not accelerate or decelerate over time [23]. In contrast, in a study of longitudinal collection of plasma NFL samples from the ADNI cohort, plasma NFL levels increased longitudinally in all diagnostic groups, with the largest increase in patients with $\mathrm{AD}$ dementia [24]. In a study analyzing the relationship between CSF and plasma biomarkers and $\mathrm{A} \beta$ positron emission tomography (PET) uptake, changes in CSF and plasma biomarkers were seen almost simultaneously. CSF NFL exhibited a sigmoid trajectory with a subtle initial increase around the same point as most other CSF biomarkers, followed by a plateau, and then a marked increase at a later stage that continued to increase throughout the span of standardized uptake value ratio (SUVR). The second increase in NFL occurred last and significantly later than that of all other biomarkers [25]. To sum up, in individuals at risk of AD, NFL levels began to increase vertically 10 years before diagnosis and con- 
tinued to increase during $\mathrm{AD}$ dementia, but it was uncertain whether the rate of NFL changed throughout the course of the disease.

\section{The NFL Levels in Familial AD}

In a cross-sectional study of PS1 or APP mutations, the authors studied 48 samples from carriers and noncarriers and reported that patients with familial $\mathrm{AD}$ had elevated serum NFL concentrations before the onset of symptoms [26]. Further research by the same team showed that this increase in serum NFL concentration occurred for the first time more than a decade before the onset of clinical symptoms and continued to rise after that [27]. In a Spanish cohort, NFL was measured cross-sectionally in 60 individuals from autosomal-dominant Alzheimer's disease families and longitudinally in an exploratory study in a subset of 6 mutation carriers. It was found that the annual increase rate of symptomatic mutation carriers was higher than that of asymptomatic mutation carriers. It is speculated that in the asymptomatic stage of the disease, neurodegeneration may accelerate close to the onset of symptoms. At the same time, we should also note that the lack of repeated individual samples in most of the participants also limits the interpretation of the reliability of the marker at the individual level in longitudinal studies [28]. Another longitudinal study of serum NFL also confirmed that the change rate of serum NFL reached a peak when participants switched from presymptomatic to symptomatic phase and reached a plateau during the symptomatic phase, that is, the absolute value of NFL tended to increase slowly over time. The change rate of serum NFL can distinguish between mutation carriers and nonmutation carriers nearly 10 years earlier than the cross-sectional absolute NFL level (i.e., 16.2 vs. 6.8 years before the onset of symptoms). In other words, subtle longitudinal changes with the NFL can be detected 16 years before the onset of cognitive symptoms [29]. Therefore, in familial $\mathrm{AD}$, from 16 years before the onset of symptoms to the onset of symptoms, the serum NFL has been increasing longitudinally, and the change in NFL level will accelerate or decelerate with the passage of time. The familial Alzheimer's disease Colombian kindred aged 8-75 years with no other neurological or health conditions from the Alzheimer's Prevention Initiative Registry at the University of Antioquia (Medellin, Colombia) were recruited in the study, and a baseline assessment was conducted among 1,070 carriers of PSEN1E280A mutation and 1,074 noncarriers. Of these participants, $242 \mathrm{mu}$ tation carriers and 262 noncarriers were measured longitudinally (with a mean follow-up of 6 years). The study

The Potential of NFL as an AD Biomarker found that baseline and longitudinal plasma NFL measurements differed significantly in impaired carriers, unimpaired carriers, and noncarriers and increased with age in all groups. Also, it began to differentiate carriers from noncarriers when aged 22 years (22 years before the estimated median age at MCI onset of 44 years), although the ability of plasma NFL to discriminate between carriers and noncarriers only reached high sensitivity close to the age of clinical onset [30].

\section{The Potential of NFL as a Biomarker in AD}

\section{Relationship between NFL and Age, Sex, and Biomarkers \\ Age}

CSF NFL levels increase with age [7, 17, 22, 31, 32]. Similarly, plasma NFL levels are also significantly related to age $[23,31,33]$. It is speculated that this may be due to age-related atrophy changes or insufficient brain metabolism, resulting in axonal degeneration and more NFL release [34]. Therefore, age effect should be taken into account when analyzing the concentration of NFL.

\section{Gender}

Most studies suggest that plasma NFL levels are not related to sex $[17,24,31,35,36]$. However, there are studies that emphasize the importance of gender-based adjustment. Two cross-sectional studies have found that male patients had higher plasma NFL levels than female patients $[32,34]$, equivalent to an age increase of 7.6 years [32]. Another study found that women with normal cognition had higher levels of CSF NFL [31]. The relationship between NFL levels and gender has not been consistently observed, and additional research is needed to determine what causes or lacks gender differences.

\section{Genetic Factors}

Plasma NFL levels have nothing to do with apolipoprotein E $\varepsilon 4$ genotype [22, 34-36]. In familial AD, adjusting for age, gender, and estimated number of years of symptoms (EYO), there was no significant difference in NFL values between PS1 carriers and APP carriers. In PS1, there was no significant difference in NFL concentration before and after mutation of codon 200 [27].

\section{Relationship with AD Core Biomarkers}

Amyloid $\beta$

Some studies have suggested that the CSF NFL level has nothing to do with $A \beta[7,24,37]$. CSF NFL concen- 
trations are not driven by pathological A $\beta 42$ [38]. NFLrelated variables have no different amyloid status [32]. However, a longitudinal study also showed that CSF NFL is related to preclinical $A \beta$, which included 770 individuals from the EMIF-AD Multimodal Biomarker Discovery study, and found that in $A \beta+$ individuals, the NFL levels of the normal cognitive group have increased, while in $\mathrm{A} \beta$ - individuals, NFL levels only increase from the MCI stage [39].

In a cross-sectional study of $\mathrm{AD}$ mutation carriers, there was no correlation between serum NFL and CSF A $\beta 42$ levels [28]. However, in a longitudinal study, the correlation between the change rate of plasma NFL and the longitudinal data of other biomarkers in $243 \mathrm{CN}$ participants from the ADNI database was found by using the multivariate linear mixed-effect model to test the correlation between plasma NFL change rate and other biomarkers. There was a correlation between the estimated annual rate of change in plasma NFL and the estimated annual rate of change in CSF $\mathrm{A} \beta 42$ among $\mathrm{CN}$ participants with evidence of amyloid lesions $(\mathrm{CN}+)$, but there was no correlation between $\mathrm{CN}-$ participants. Therefore, in the normal cognitive period, not all individuals have reached the plateau phase of CSF $A \beta 42$ and changes in CSF A $\beta 42$ are still reflected in plasma NFL [22]. In the MCI group, plasma NFL levels are negatively correlated with CSF A $\beta 42$ levels, which support the use of plasma NFL as a sensitive biomarker for $\mathrm{AD}$-related biological changes in precursor AD [20]. Similarly, there was a similar correlation in the $\mathrm{AD}$ group [40].

In cross-sectional and longitudinal studies, the correlation between NFL and $A \beta$ seems to be inconsistent. Whether NFL is affected by pathological A $\beta 42$ needs to be confirmed in larger samples.

\section{Tau}

CSF NFL levels are associated with biomarkers of tau pathology (including T-tau and P-tau) [7, 41, 42]. Using AT(N) classification, it was found that the CSF NFL concentration of patients with positive tau pathology $(\mathrm{A}-/ \mathrm{T} \pm / \mathrm{N}+, \mathrm{A}-/ \mathrm{T}+/ \mathrm{N} \pm, \mathrm{A}+/ \mathrm{T} \pm / \mathrm{N}+$, and $\mathrm{A}+/ \mathrm{T}+/ \mathrm{N} \pm)$ was higher than that of the control group $\mathrm{AD}$-pathophysiology-negative patients $(\mathrm{A}-/ \mathrm{T}-/ \mathrm{N}-)$ and $\mathrm{A} \beta$-pathologypositive patients $(\mathrm{A}+/ \mathrm{T}-/ \mathrm{N}-)$ [43]. Longitudinal NFL levels generally increase in patients with $\mathrm{N}+$ (temporal brain atrophy as $\mathrm{N}$-classifier; $\mathrm{A}-/ \mathrm{T}+/ \mathrm{N}+, \mathrm{A}+/ \mathrm{T}+/ \mathrm{N}+$, and $\mathrm{A}+/ \mathrm{T}-/ \mathrm{N}+)$ and isolated $\mathrm{T}+(\mathrm{A}-/ \mathrm{T}+/ \mathrm{N}-)$ patients [24]. CSF NFL correlated significantly with tau in Tg4510 mice (tauopathy/AD) brains, suggesting a surrogate biomarker potential of CSF NFL, and means that CSF NFL can be used to monitor the effects of potential tau-targeted drugs in preclinical and clinical studies, but independent validation is warranted [44].

In plasma, NFL levels were also observed to be crosssectionally correlated with T-tau and P-tau [22, 28, 40], and plasma NFL levels were also correlated with plasma tau levels [20]. Retrospective stratification based on Braak staging revealed that baseline plasma NFL concentration was associated with higher postmortem neurofibrillary winding pathology [33].

\section{Accuracy of Diagnosis}

The 2018 NIA-AA Research Framework has proposed a biological definition of AD by using the AT(N) system, where $(\mathrm{N})$ is open to new biomarkers if supported by the available evidence [4]. A study has shown that NFL as a marker of neurodegenerative degeneration $(\mathrm{N})$ behaves similarly to T-tau [45]. The CSF NFL identification of AD and the control group had an area under the receiver operating characteristic curve (AUROC) of 0.84 , with a sensitivity and specificity of 81.5 and $79.7 \%$, respectively. The $\mathrm{NFL} / \mathrm{A} \beta 42$ ratio distinguished $\mathrm{AD}$ from the control group with an AUC value of 0.9 , with a sensitivity and specificity of 81.5 and $80.4 \%$, respectively. The AUC value of NFL can distinguish $\mathrm{AD}$ from the control group as effectively as CSF T-tau and A $\beta 42$, and it can distinguish the AUC value of $\mathrm{AD}$ and the control group from P-tau levels [37]. T-tau combined with NFL can improve the diagnostic accuracy of $\mathrm{AD}$ and $\mathrm{CN}$ subjects [46]. Similarly, the diagnostic accuracy of plasma NFL for AD dementia is the same as that of established CSF biomarkers [20]. (When correcting for age, sex, and educational level, the AUROCs were 0.79 for plasma NFL, 0.81 for CSF NFL, 0.85 for CSF T-tau, 0.81 for CSF P-tau, and 0.64 for plasma tau.)

\section{The Relationship between NFL and Neuroimaging}

The researchers included 88 participants in a 2 -year longitudinal study with baseline CSF measures and 2 magnetic resonance images. They found that NFL predicted hippocampal atrophy rate independently of age, $\mathrm{A} \beta 42$, and P-tau. Including NFL, P-tau, and age in the same model, higher NFL and lower P-tau predicted higher hippocampal atrophy $\left(R^{2}=0.20\right.$, NFL: $\beta=-0.34 ; p=$ 0.003 , P-tau: $\beta=0.27 ; p=0.009)$. The results were upheld in the 36 participants with very low AD risk. They suggest that factors previously shown to be important for brain degeneration in MCI may also impact changes in normal aging, demonstrating that the NFL-atrophy association is likely not caused by $\mathrm{AD}$-specific mechanisms, and it is 
likely to indicate $\mathrm{AD}$-independent, age-expected neurodegeneration [42]. In a longitudinal study of 221 participants from the Australian Imaging, Biomarkers \& Lifestyle Flagship Study of Aging, the researchers reported that there was a significant negative moderate correlation between cortical gray matter volume and NFL ( $r=-0.38$, $p<0.001)$ and a significant weak negative correlation between hippocampal volume and NFL $(r=-0.27, p<$ $0.001)$. CSF NFL levels were not associated significantly with either white matter volume $(r=0.03, p=0.687)$ or ventricular volume $(r=0.104, p=0.166)$. Because there was a significant moderate correlation between CSF NFL and cortical gray matter volume, the utility of CSF NFL as a predictor of baseline gray matter volume was assessed further. CSF NFL $(\beta=-10.15$, standardized $\beta=-0.29$, $p<0.001)$ and NFL/A $\beta 42$ ratio $(\beta=-6.99$, standardized $\beta=-0.43, p<0.001)$ correlated significantly with smaller baseline cortical gray matter volume. The ratio of CSF NFL and A $\beta 42$ levels $\left(R^{2}=32 \%\right)$ was a better predictor of cortical brain atrophy than either of these measures individually. Therefore, CSF NFL should be used in combination with these more disease-specific biomarkers, as it is a general biomarker of neurodegeneration and not specific to any particular neurodegenerative disease [37].

To study the relationship between plasma and CSF NFL and longitudinal neuroimaging, the researchers recruited 79 nondementia subjects with an average age of 76 years from the Mayo Clinic Study of Aging. They found there was no cross-sectional association between NFL in CSF or plasma and any neuroimaging in subjects with normal cognitive function. Longitudinally, higher baseline CSF NFL was associated with worsening in cortical thickness and fractional anisotropy (FA) of the corpus callosum, and higher baseline plasma NFL was associated with worsening in all neuroimaging measures, except amyloid PET (including hippocampal volume, cortical thickness, fluorodeoxyglucose PET (FDG-PET), and corpus callosum FA). The increase in plasma NFL levels during short-term follow-up is associated with increased $\mathrm{A} \beta \mathrm{li}$ gand retention on PET, which may reflect ongoing $\mathrm{AD}$ associated neurodegeneration. However, compared with CSF NFL, more statistically significant associations were found for plasma NFL for neuroimaging. There are a few explanations as to why they may have obtained this result. First, the researchers used different assays for plasma and CSF because of the difference in concentrations between the 2 mediums. Thus, it is possible that there was more variability or other differences in the CSF assay compared to the plasma assay. Second, the sample size of the study is very small, which may lead to false-positive results for plasma NFL and false-negative results for CSF NFL. Additional longitudinal studies measuring CSF and plasma NFL as well as larger sample sizes are needed to validate these conclusions. Finally, there may be different changes in CSF and plasma NFL during clinical progression, similar to the observation that CSF A $\beta 42$ changes earlier than amyloid PET. A series of assessments of plasma and CSF NFL are needed to determine whether these markers are related to clinical progression [36].

Several studies from the ADNI cohort described in detail the relationship between NFL and brain structural changes in $\mathrm{AD}$. In the $\mathrm{MCI}$ group and $\mathrm{AD}$ dementia group with $A \beta$ pathological features, high plasma NFL levels were associated with atrophy of AD-related areas and in brain hypometabolic areas at baseline and longitudinal changes [20]. Further study found that the baseline plasma NFL levels in patients with MCI were associated with low metabolism in bilateral parahippocampal and middle temporal gyrus, and the high levels of NFL in CSF were associated with bilateral anterior and posterior cingulate cortex hypometabolism. In the multiple regression model with age, sex, cognitive score, and plasma tau level as covariables, high baseline plasma NFL levels in patients with MCI could independently predict cortical hypometabolism in bilateral parahippocampal gyrus, right fusiform gyrus, and middle temporal gyrus [47].

A recent study also confirmed that CSF and plasma NFL levels are associated with brain hypometabolism in $\mathrm{AD}$ marker areas. The subjects were from the ADNI cohort. The cross-sectional analysis included 243 participants, and the longitudinal part included 1,015 participants (the average follow-up time was 59.2 months). Compared with $\mathrm{A} \beta$ negative, the correlation between increased plasma NFL concentration and decreased glucose metabolism was stronger in patients with cognitive impairment who were $A \beta$ positive. The longitudinal correlation between plasma NFL and brain metabolic changes over 24 months existed only in $\mathrm{A} \beta$-positive individuals. In $A \beta$-positive subjects, plasma NFL is a marker of neurodegeneration in areas associated with $\mathrm{AD}$ [31]. To study the relationship between longitudinal changes in plasma NFL levels and changes in other indicators, a study collected plasma samples over a period of 11 years and found that longitudinal increases in plasma NFL levels were associated with baseline MRI manifestations (small hippocampal volume, thin regional cortex, and large ventricular volume) and low FDG-PET uptake. The increasing rate of NFL levels was positively correlated with the rate of brain atrophy and metabolic decline, especially in patients with MCI [24]. It is worth noting that 
they did not control for AD phenotype subtypes and they cannot exclude the possibility that comorbidities (including $\mathrm{VaD}$ ) contributed to the results. There is a great deal of variability between subjects as compared to more widely used neurodegeneration biomarkers such as MRI or FDG-PET.

In patients with familial AD with PS1 or APP mutations, serum NFL is associated with brain volume loss and longitudinal brain atrophy, suggesting that serum NFL concentration may be related to the severity of the disease or the rate of progression. However, there was no significant correlation between serum NFL and hippocampal volume, indicating that the elevated serum NFL levels more closely reflect global neurodegeneration. The correlation between focal atrophy and NFL seems to be stronger than that of whole brain atrophy in neurodegenerative diseases such as progressive supranuclear paralysis (PSP) and FTD. It may therefore be that serum NFL will be most useful for identifying and tracking AD-related neurodegeneration when combined with a test to confirm underlying $\mathrm{AD}$ molecular pathology, that is, CSF tau/A $\beta 1-42$ or amyloid PET [26]. Another study has shown that the change rate of serum NFL is associated with cortical thinning assessed by MRI, but not with $A \beta$ deposition or glucose metabolism (assessed by PET). Serum NFL can predict the rate of cerebral cortex thinning [29].

\section{The Relationship between NFL and Cognition}

The relationship between NFL and cognitive performance has attracted more and more attention. In a recent metaregression analysis, it was found that there was a significant negative correlation between NFL levels and MMSE scores [7].

In the middle-aged and elderly cohort of cognitive health, the levels of NFL in CSF were not related to the cognitive ability at baseline [48]. Compared with neurogranin, chitinase 3 -like protein 1 , and $\mathrm{T}$-tau, high baseline NFL levels predicted cognitive decline in $\mathrm{A} \beta$-positive individuals with $\mathrm{CN}, \mathrm{MCI}$, and $\mathrm{AD}$-type dementia, independent of the other markers. Indicating NFL was the better predictor of cognitive decline in $\mathrm{A} \beta$-positive individuals across the cognitive spectrum [39]. In the MCI group, CSF NFL levels were associated with cognitive deterioration during the follow-up period [38]. In another case-control study, the level of CSF NFL was positively correlated with the decreasing rate of MMSE in the AD group [19]. These studies support the use of NFL concentration in CSF as a marker for the progression of MCI and $\mathrm{AD}$ and suggest that the degeneration of large-cali- ber axons is an important factor in the progression of $\mathrm{AD}$. The diagnostic value of CSF NFL concentration may be limited due to overlap with other neurodegenerative diseases, but high levels of NFL in CSF of AD or MCI suggest that rapid progression of the disease can be expected [38]. Most studies have focused on a holistic cognitive measurement as the main result, and it is not clear whether the rate of decline caused by NFL varies from region to region. In the middle-aged and elderly cohort with cognitive health, a total of 255 participants completed CSF studies and serial cognitive assessments to measure global cognition, episodic memory, and attentional control. They concluded that CSF NFL can predict the decline of overall cognitive ability and attention, but cannot predict the decline of episodic memory, which has nothing to do with the status of amyloid protein. However, an episodic memory composite may be more specific to $\mathrm{AD}$-specific neurodegeneration (i.e., only among individuals who are positive for amyloid). The finding that NFL does not interact with amyloid in our statistical models may indicate that NFL is capturing both AD-related pathology as well as unrelated neurodegeneration. It is important to account for these extraneous neurodegenerative processes when building a model of cognitive function in $\mathrm{AD}$ [48].

Similarly, in individuals with normal cognition, plasma NFL was not related to MMSE score [17]. In the MCI group and AD dementia group, plasma NFL was correlated with global cognition and executive function at baseline, and NFL was more correlated with timing tests (including the Trail-Making Test part B and Wechsler Adult Intelligence Scale-Revised digit symbol substitution tests) than memory tests. Longitudinally, the only statistically significant interaction was for the MCI group and MMSE score, demonstrating that plasma NFL levels were more strongly correlated with longitudinal MMSE scores in the MCI group than in controls. It is suggested that plasma NFL mainly reflects the damage to the larger myelinated axons of neurons [20]. A cross-sectional study of 48 people recruited from families with PSEN1 or APP mutations found that there was a stronger correlation between serum NFL and overall cognitive measurements than with memory scores, suggesting that the initial increase may reflect the subtle widespread collapse of the neural network rather than focal hippocampal (gray matter) atrophy, in contrast to the findings of other neurodegenerative diseases, including PSP and FTD. The correlation between focal atrophy and NFL in these diseases seems to be stronger than that of global brain atrophy [26].
12

Eur Neurol 2021;84:6-15 DOI: $10.1159 / 000513008$
Xiong/Meng/Luo/Zhang 
Baseline plasma NFL can predict cognitive impairment, especially in preclinical AD patients. The faster the plasma NFL level increased, the faster the overall cognitive ability deteriorated, and this correlation was most significant in the MCI group [24]. Plasma NFL shows a special prospect as an available biomarker related to cognition in MCI [49]. One study suggested that the change rate of plasma NFL had no predictive effect on cognitive impairment in nondementia elderly [22]. It has also been suggested that although prospective predictive analysis is not sufficient to prove that the rate of change of NFL is indeed a better predictor of disease progression (neurodegeneration and cognitive decline) than the absolute level of NFL, it is based on 2 points: first, patients with sporadic $\mathrm{AD}$ are usually older and have more comorbidities, which in turn may affect plasma NFL levels; second, compared with the absolute level of NFL, the rate of change of NFL is a better predictor of AD before symptoms, so in a certain period of the disease, the rate of change of NFL is more likely to predict cognitive decline than absolute NFL, but future analysis should evaluate this period more accurately [29].

\section{Conclusions and Future Aspects}

The level of NFL is elevated in many neurodegenerative diseases and is not disease-specific, so it is not suitable to be used as a diagnostic marker of $\mathrm{AD}$, but its value in judging the severity of the disease and predicting disease progression and prognosis cannot be ignored [4]. In $\mathrm{AD}$, NFL levels begin to rise more than a decade before symptoms appear and can predict cognitive impairment, atrophy of AD-related areas, and longitudinal changes in areas of the brain with low metabolism. In preclinical AD patients, the correlation between NFL and core markers, neuroimaging, and cognitive changes is particularly significant. Based on individual differences such as age and codisease, some studies have suggested that the rate of change of NFL may better reflect the potential of NFL as a biomarker in AD than the absolute value of NFL. Therefore, future studies need to focus on how NFL changes throughout the course of the disease and whether the absolute level of NFL or the rate of change of NFL at each stage of the disease is more likely to predict the progression and severity of the disease. It is worth noting that one of the limitations of NFL is that there is a great deal of variability between subjects as compared to more widely used neurodegeneration biomarkers such as MRI or FDG-PET; thus, more studies involving a variety of neurodegenerative diseases are warranted to investigate the roles of plasma NFL. In addition, through the GWAS, it was found that SNPs at multiple loci affected NFL levels and AD risk, but there were few studies on genetic variation, so we need to verify these findings in different populations and look for new variants.

\section{Statement of Ethics}

The authors have no ethical conflicts to declare.

\section{Conflict of Interest Statement}

The authors have no conflicts of interest to declare.

\section{Funding Sources}

This study was supported by the Medical Research Project of Chongqing Healthy Committee (2018MSXM058).

\section{Author Contributions}

Y.X. conceived the project, carried out analysis of previous topics, and wrote the preliminary draft. T.M. and J.L. conceived the project. H.Z. contributed to the final preparation of the manuscript and supervised the project. All authors contributed to the final draft of manuscript.

\section{References}

1 Petzold A. Neurofilament phosphoforms: surrogate markers for axonal injury, degeneration and loss. J Neurol Sci. 2005 Jun 15; 233(1-2):183-98.

2 Gaiottino J, Norgren N, Dobson R, Topping J, Nissim A, Malaspina A, et al. Increased neurofilament light chain blood levels in neurodegenerative neurological diseases. PLoS One. 2013;8(9):e75091.

The Potential of NFL as an AD Biomarker
3 Zhao Y, Xin Y, Meng S, He Z, Hu W. Neurofilament light chain protein in neurodegenerative dementia: a systematic review and network meta-analysis. Neurosci Biobehav Rev. 2019 Jul;102:123-38.

4 Jack CR Jr, Bennett DA, Blennow K, Carrillo MC, Dunn B, Haeberlein SB, et al. NIA-AA Research Framework: toward a biological definition of Alzheimer's disease. Alzheimers Dement. 2018 Apr;14(4):535-62.
5 Skillback T, Farahmand B, Bartlett JW, Rosen C, Mattsson N, Nagga K, et al. CSF neurofilament light differs in neurodegenerative diseases and predicts severity and survival. Neurology. 2014 Nov 18;83(21):1945-53.

6 Bacioglu M, Maia LF, Preische O, Schelle J, Apel A, Kaeser SA, et al. Neurofilament light chain in blood and CSF as marker of disease progression in mouse models and in neurodegenerative diseases. Neuron. 2016 Jul 6;91(2):494-6. 
7 Jin M, Cao L, Dai YP. Role of neurofilament light chain as a potential biomarker for Alzheimer's disease: a correlative meta-analysis. Front Aging Neurosci. 2019;11:254.

8 Kuhle J, Barro C, Andreasson U, Derfuss T, Lindberg R, Sandelius Å, et al. Comparison of three analytical platforms for quantification of the neurofilament light chain in blood samples: ELISA, electrochemiluminescence immunoassay and SIMOA. Clin Chem Lab Med. 2016 Oct 1;54(10):1655-61.

9 Niikado M, Chrem-Méndez P, Itzcovich T, Barbieri-Kennedy M, Calandri I, Martinetto $\mathrm{H}$, et al. Evaluation of cerebrospinal fluid neurofilament light chain as a routine biomarker in a memory clinic. J Gerontol A Biol Sci Med Sci. 2019 Mar 14;74(4):442-5.

10 Fuchs E, Cleveland DW. A structural scaffolding of intermediate filaments in health and disease. Science. 1998 Jan 23;279(5350): $514-9$.

11 Rosengren LE, Karlsson JE, Karlsson JO, Persson LI, Wikkelsø C. Patients with amyotrophic lateral sclerosis and other neurodegenerative diseases have increased levels of neurofilament protein in CSF. J Neurochem. 1996 Nov;67(5):2013-8.

12 Fernandez-Martos CM, King AE, Atkinson RA, Woodhouse A, Vickers JC. Neurofilament light gene deletion exacerbates amyloid, dystrophic neurite, and synaptic pathology in the APP/PS1 transgenic model of Alzheimer's disease. Neurobiol Aging. 2015 Oct;36(10): 2757-67.

13 Vickers JC, King AE, McCormack GH, Bindoff $\mathrm{AD}$, Adlard PA. Iron is increased in the brains of ageing mice lacking the neurofilament light gene. PLoS One. 2019;14(10): e0224169.

14 Niu L-D, Xu W, Xu W, Li J-Q, Tan C-C, Cao $\mathrm{X}-\mathrm{P}$, et al. Genome-wide association study of cerebrospinal fluid neurofilament light levels in non-demented elders. Ann Transl Med. 2019 Nov;7(22):657.

15 Li JQ, Yuan XZ, Li HY, Cao XP, Yu JT, Tan L, et al. Genome-wide association study identifies two loci influencing plasma neurofilament light levels. BMC Med Genomics. 2018 May 10;11(1):47.

16 Wang Z-T, Chen S-D, Xu W, Chen K-L, Wang H-F, Tan C-C, et al. Genome-wide association study identifies CD1A associated with rate of increase in plasma neurofilament light in non-demented elders. Aging. $2019 \mathrm{Jul}$ 11;11(13):4521-35.

17 Zhou W, Zhang J, Ye F, Xu G, Su H, Su Y, et al. Plasma neurofilament light chain levels in Alzheimer's disease. Neurosci Lett. 2017 May 22;650:60-4.

18 Lewczuk P, Ermann N, Andreasson U, Schultheis C, Podhorna J, Spitzer P, et al. Plasma neurofilament light as a potential biomarker of neurodegeneration in Alzheimer's disease. Alzheimers Res Ther. 2018 Jul 28;10(1):71.
19 Olsson B, Portelius E, Cullen NC, Sandelius $\AA$ Å, Zetterberg H, Andreasson U, et al. Association of cerebrospinal fluid neurofilament light protein levels with cognition in patients with dementia, motor neuron disease, and movement disorders. JAMA Neurol. 2019 Mar 1;76(3):318-25.

20 Mattsson N, Andreasson U, Zetterberg H, Blennow K; Alzheimer's Disease Neuroimaging Initiative. Association of plasma neurofilament light with neurodegeneration in $\mathrm{pa}$ tients with Alzheimer disease. JAMA Neurol. 2017 May 1;74(5):557-66.

21 Chatterjee P, Goozee K, Sohrabi HR, Shen K, Shah T, Asih PR, et al. Association of plasma neurofilament light chain with neocortical amyloid- $\beta$ load and cognitive performance in cognitively normal elderly participants. J Alzheimers Dis. 2018;63(2):479-87.

$22 \mathrm{Hu} \mathrm{H}$, Chen K-L, Ou Y-N, Cao X-P, Chen $S-D$, Cui M, et al. Neurofilament light chain plasma concentration predicts neurodegeneration and clinical progression in nondemented elderly adults. Aging. 2019 Sep 12; 11(17):6904-14.

23 de Wolf F, Ghanbari M, Licher S, McRaeMcKee K, Gras L, Weverling GJ, et al. Plasma tau, neurofilament light chain and amyloid- $\beta$ levels and risk of dementia; a populationbased cohort study. Brain. 2020 Apr 1;143(4): 1220-32.

24 Mattsson N, Cullen NC, Andreasson U, Zetterberg H, Blennow K. Association between longitudinal plasma neurofilament light and neurodegeneration in patients with Alzheimer disease. JAMA Neurol. 2019 Jul 1; 76(7):791-9.

25 Palmqvist S, Insel PS, Stomrud E, Janelidze S, Zetterberg H, Brix B, et al. Cerebrospinal fluid and plasma biomarker trajectories with increasing amyloid deposition in Alzheimer's disease. EMBO Mol Med. 2019 Dec;11(12): e11170.

26 Weston PSJ, Poole T, Ryan NS, Nair A, Liang $Y$, Macpherson K, et al. Serum neurofilament light in familial Alzheimer disease: a marker of early neurodegeneration. Neurology. 2017 Nov 21;89(21):2167-75

27 Weston PSJ, Poole T, O'Connor A, Heslegrave A, Ryan NS, Liang Y, et al. Longitudinal measurement of serum neurofilament light in presymptomatic familial Alzheimer's disease. Alz Res Therapy. 2019 Feb 20;11(1):19.

28 Sanchez-Valle R, Heslegrave A, Foiani MS, Bosch B, Antonell A, Balasa M, et al. Serum neurofilament light levels correlate with severity measures and neurodegeneration markers in autosomal dominant Alzheimer's disease. Alzheimers Res Ther. 2018 Nov 3; 10(1):113

29 Preische O, Schultz SA, Apel A, Kuhle J, Kaeser SA, Barro C, et al. Serum neurofilament dynamics predicts neurodegeneration and clinical progression in presymptomatic $\mathrm{Al}$ zheimer's disease. Nat Med. 2019 Feb;25(2): 277-83.
30 Quiroz YT, Zetterberg H, Reiman EM, Chen Y, Su Y, Fox-Fuller JT, et al. Plasma neurofilament light chain in the presenilin $1 \mathrm{E} 280 \mathrm{~A}$ autosomal dominant Alzheimer's disease kindred: a cross-sectional and longitudinal cohort study. Lancet Neurol. 2020;19(6): 513-21.

31 Benedet AL, Ashton NJ, Pascoal TA, Leuzy A, Mathotaarachchi S, Kang MS, et al. Plasma neurofilament light associates with Alzheimer's disease metabolic decline in amyloid-positive individuals. Alzheimers Dement. 2019 Dec;11:679-89.

32 Mielke MM, Syrjanen JA, Blennow K, Zetterberg H, Skoog I, Vemuri P, et al. Comparison of variables associated with cerebrospinal fluid neurofilament, total-tau, and neurogranin. Alzheimers Dement. 2019 Nov;15(11):143747.

33 Ashton NJ, Leuzy A, Lim YM, Troakes C, Hortobágyi T, Höglund $\mathrm{K}$, et al. Increased plasma neurofilament light chain concentration correlates with severity of post-mortem neurofibrillary tangle pathology and neurodegeneration. Acta Neuropathol Commun. 2019 Jan 9;7(1):5

34 Lin YS, Lee WJ, Wang SJ, Fuh JL. Levels of plasma neurofilament light chain and cognitive function in patients with Alzheimer or Parkinson disease. Sci Rep. 2018 Nov 26;8(1): 17368.

35 Lleo A, Alcolea D, Martinez-Lage P, Scheltens P, Parnetti L, Poirier J, et al. Longitudinal cerebrospinal fluid biomarker trajectories along the Alzheimer's disease continuum in the BIOMARKAPD study. Alzheimers Dement. 2019 Jun;15(6):742-53.

36 Mielke MM, Syrjanen JA, Blennow K, Zetterberg H, Vemuri P, Skoog I, et al. Plasma and CSF neurofilament light: relation to longitudinal neuroimaging and cognitive measures. Neurology. 2019 Jul 16;93(3):e252-60.

37 Dhiman K, Gupta VB, Villemagne VL, Eratne D, Graham PL, Fowler C, et al. Cerebrospinal fluid neurofilament light concentration predicts brain atrophy and cognition in Alzheimer's disease. Alzheimers Dement. 2020; 12(1):e12005

38 Zetterberg H, Skillbäck T, Mattsson N, Trojanowski JQ, Portelius E, Shaw LM, et al. Association of cerebrospinal fluid neurofilament light concentration with Alzheimer disease progression. JAMA Neurol. 2016 Jan; 73(1):60-7.

39 Bos I, Vos S, Verhey F, Scheltens P, Teunissen C, Engelborghs S, et al. Cerebrospinal fluid biomarkers of neurodegeneration, synaptic integrity, and astroglial activation across the clinical Alzheimer's disease spectrum. Alzheimers Dement. 2019 May;15(5):644-54.

40 Liu S, Huang Z, Zhang L, Pan J, Lei Q, Meng $\mathrm{Y}$, et al. Plasma neurofilament light chain may be a biomarker for the inverse association between cancers and neurodegenerative diseases. Front Aging Neurosci. 2020;12:10. 
41 Melah KE, Lu SY, Hoscheidt SM, Alexander AL, Adluru N, Destiche DJ, et al. Cerebrospinal fluid markers of Alzheimer's disease pathology and microglial activation are associated with altered white matter microstructure in asymptomatic adults at risk for Alzheimer's disease. J Alzheimers Dis. 2016;50(3):873-86.

42 Idland AV, Sala-Llonch R, Borza T, Watne LO, Wyller TB, Brækhus A, et al. CSF neurofilament light levels predict hippocampal atrophy in cognitively healthy older adults. Neurobiol Aging. 2017 Jan;49:138-44.

43 Lista S, Toschi N, Baldacci F, Zetterberg H, Blennow K, Kilimann I, et al. Diagnostic accuracy of CSF neurofilament light chain protein in the biomarker-guided classification system for Alzheimer's disease. Neurochem Int. 2017 Sep;108:355-60.
44 Clement A, Mitchelmore C, Andersson DR, Asuni AA. Cerebrospinal fluid neurofilament light chain as a biomarker of neurodegeneration in the Tg4510 and MitoPark mouse models. Neuroscience. 2017 Jun 23;354:101-9.

45 Antonell A, Tort-Merino A, Ríos J, Balasa M, Borrego-Écija S, Auge JM, et al. Synaptic, axonal damage and inflammatory cerebrospinal fluid biomarkers in neurodegenerative dementias. Alzheimers Dement. 2020 Feb;16(2): 262-72.

46 Mattsson N, Insel PS, Palmqvist S, Portelius E, Zetterberg $\mathrm{H}$, Weiner $\mathrm{M}$, et al. Cerebrospinal fluid tau, neurogranin, and neurofilament light in Alzheimer's disease. EMBO Mol Med. 2016 Oct;8(10):1184-96.
47 Mayeli M, Mirshahvalad SM, Aghamollaii V, Tafakhori A, Abdolalizadeh A, Rahmani F. Plasma neurofilament light chain levels are associated with cortical hypometabolism in Alzheimer disease signature regions. J Neuropathol Exp Neurol. 2019 Jul 15.

48 Aschenbrenner AJ, Gordon BA, Fagan AM, Schindler SE, Balota DA, Morris JC, et al. Neurofilament light predicts decline in attention but not episodic memory in preclinical Alzheimer's disease. J Alzheimers Dis. 2020; 74(4):1119-29.

49 Osborn KE, Khan OA, Kresge HA, Bown CW, Liu D, Moore EE, et al. Cerebrospinal fluid and plasma neurofilament light relate to abnormal cognition. Alzheimers Dement. 2019 Dec;11:700-9. 